\title{
MEMPHA: Model of Exascale message-passing programs on heterogeneous architectures
}

\begin{abstract}
Delivering optimum performance on a parallel computer is highly dependant on the efficiency of the scheduling and mapping procedure. If the composition of the parallel application is known a prior, the mapping can be accomplished statically on the compilation time. The mapping algorithm uses the model of the parallel application and maps its tasks to processors in a way to minimize the total execution time. In this article, current modeling approaches have discussed. Later, a new modeling schema named Model of Exascale Message-Passing Programs on Heterogeneous Architectures (MEMPHA) has proposed. A comparative study has been performed between MEMPHA and existing models. To exhibit the efficiency of the MEMPHA, experiments have performed on a set of data-set hypergraphs. The results obtained from the experiments show that deploying the MEMPHA helps to optimize metrics, including the congestion, total communication volume and maximum volume of data being sent or received. These improvements vary from 76 to 1 percent, depending on the metric and benchmark model. Moreover, MEMPHA supports the modeling of applications with multiple producers for a single data transmission, where the rest of the approaches fail.
\end{abstract}

Keyword: Parallel models; Scheduling and task partitioning; Heterogeneous (hybrid) systems; Modelling and prediction 